\title{
Synthesis of 5,8- and 5,6-Quinolinediones Using Oxidative Demethylation with Cerium(IV) Ammonium Nitrate
}

\author{
Yoshiyasu Kitahara, Masanori Nagatsu, Yoshikazu Shibano, and Akinori Kubo* \\ Meiji College of Pharmacy, 1-35-23 Nozawa, Setagaya-ku, Tokyo 154, Japan. \\ Received April 17, 1997; accepted June 9, 1997
}

\begin{abstract}
4-Phenyl-5,8-quinolinediones $(7,8,12,13)$, 4-phenyl-5,6-quinolinediones $(9,14)$, and 2-dialkylamino-4-phenyl5,8 -quinolinediones $(17,18)$, were synthesized by oxidative demethylation of the corresponding 5,8-dimethoxy- or 5,6,8-trimethoxy-4-phenylquinolines with cerium(IV) ammonium nitrate. Sulfur-containing quinolinequinones (21, 24) were prepared by oxidation of the corresponding 5,8-dimethoxy- or 5,6,8-trimethoxy-2(1H)-quinolinethiones $(20,23)$.
\end{abstract}

Key words quinolinequinone; oxidative demethylation; cerium(IV) ammonium nitrate

Streptonigrin, a highly substituted 5,8-quinolinedione, is one of the most potent inhibitors of avian myeloblastosis virus reverse transcriptase (AMV-RT), and the 7-amino6-methoxy-5,8-quinolinedione moiety of streptonigrin is the minimum structure for inhibition of AMV-RT. ${ }^{1)}$ Reverse transcriptase is considered to be an excellent target for the chemotherapy of retroviral diseases, such as human acquired immunodeficiency syndrome (AIDS). We observed that 6-methoxy-5,8-quinolinediones and 8methoxy-5,6-quinolinediones were as potent (as inhibitors of AMV-RT) as streptonigrin, and much less toxic. ${ }^{2}$ We also prepared various quinolinequinones, $2(1 \mathrm{H})$-quinolinonequinones, and isoquinolinequinones by oxidative demethylation of the corresponding 5,8-dimethoxy- or $5,6,8$-trimethoxy(iso)quinolines with cerium(IV) ammonium nitrate (CAN) or silver(II) oxide, and examined their inhibitory activities. ${ }^{2 b, 3)}$ Here we report the synthesis of 4-phenylquinolinequinones and sulfur-containing quinolinequinones by oxidative demethylation of 4-phenylquinolines and $2(1 H)$-quinolinethiones.

4-Phenyl-5,8-quinolinediones $(7,8,12,13,17,18)$ and 4-phenyl-5,6-quinolinediones $(\mathbf{9}, \mathbf{1 4})$ were prepared from 4-phenyl-2 $(1 H)$-quinolinones $(\mathbf{1}, \mathbf{2}){ }^{3 c)}$ 4-Phenyl-2-trifluoromethanesulfonyloxyquinolines $(3,4)$, prepared from $2(1 H)$-quinolinones $(\mathbf{1}, \mathbf{2})$, were reduced with triethylammonium formate in the presence of palladium acetate and $1,1^{\prime}$-bis(diphenylphosphino)ferrocene (DPPF) ${ }^{4}$ to afford the 2-unsubstituted quinolines $(\mathbf{5}, \mathbf{6})$ in $88-99 \%$ yields. 2-Chloro-4-phenylquinolines $(\mathbf{1 0}, \mathbf{1 1})$ prepared by chlorination of the $2(1 H)$-quinolinones $(\mathbf{1}, \mathbf{2})$ with phosphorus oxychloride, were treated with cyclic secondary amines (piperidine, pyrrolidine, morpholine, 1-methylpiperazine) to afford 2-dialkylaminoquinolines $(\mathbf{1 5}, \mathbf{1 6})$ in $94-99 \%$ yields.

Oxidative demethylation of 5,8-dimethoxy-4-phenylquinolines $(5,10,15)$ with $C A N$ in acetonitrile-water at $0-5^{\circ} \mathrm{C}$ furnished the corresponding $p$-quinones $(\mathbf{7}, \mathbf{1 2}$, 17) in $45-94 \%$ yields. Treatment of 5,6,8-trimethoxy-4phenylquinolines $(\mathbf{6}, \mathbf{1 1})$ with CAN furnished $p$-quinones $(8,13 ; 24-33 \%$ yields) and $o$-quinones $(9,14 ; 24-75 \%$ yields). In contrast, 2-dialkylamino-5,6,8-trimethoxy-4phenylquinolines (16) were oxidized with CAN to furnish exclusively $p$-quinones (18) in $43-87 \%$ yields.

Sulfur-containing quinolinequinones $(\mathbf{2 1}, \mathbf{2 4})$ were pre- pared from $2(1 H)$-quinolinones $(\mathbf{1 9}, \mathbf{2 2}) .^{3 c)}$ Treatment of 19 (or 22) with Lawesson's reagent (2,4-bis(4-methoxyphenyl)-1,3-dithia-2,4-diphosphetane-2,4-disulfide) in 1,2dimethoxyethane afforded the corresponding $2(1 \mathrm{H})$-quinolinethiones $(\mathbf{2 0}, \mathbf{2 3})$ in $91-99 \%$ yields. Oxidative demethylation of $N$-unsubstituted $2(1 \mathrm{H})$-quinolinethiones (20) with CAN in acetonitrile-water at $0-5^{\circ} \mathrm{C}$ furnished "dimeric" quinones (21) in 56-76\% yields. The $N$-methyl$2(1 H)$-quinolinethione (23a) was oxidatively demethylated with CAN to afford the expected quinone (24a) in 15\% yield. However, attempted oxidation of 23b with CAN failed, giving a complex mixture. The quinone (24b) was obtained by oxidative demethylation of $\mathbf{2 3 b}$ with silver(II) oxide-nitric acid ${ }^{5)}$ in $49 \%$ yield.

Analytical and spectral data for the quinones (7-9, $12-14,17,18,21,24)$ are given in Table I. These quinones showed no activity against human immunodeficiency virus.

\section{Experimental}

All melting points were determined on a Yanagimoto micromelting point apparatus and are uncorrected. ${ }^{1} \mathrm{H}$-NMR spectra were measured at $270 \mathrm{MHz}$ in $\mathrm{CDCl}_{3}$ with tetramethylsilane as an internal standard. All reactions were run with magnetic stirring. Anhydrous sodium sulfate was used for drying organic solvent extracts, and the solvent was removed with a rotary evaporator and finally under high vacuum. Column chromatography (flash chromatography) was performed with Silica gel 60 (230- 400 mesh).

2-Trifluoromethanesulfonyloxy-4-phenylquinolines $(3,4)$ Trifluoromethanesulfonic anhydride $(0.1 \mathrm{ml}, 0.6 \mathrm{mmol})$ was added to a solution of 4-phenyl-2(1 H)-quinolinone $(\mathbf{1}, \mathbf{2})(0.5 \mathrm{mmol})$ in dry $\mathrm{CH}_{2} \mathrm{Cl}_{2}(10 \mathrm{ml})$ containing triethylamine $(0.2 \mathrm{ml}, 1.4 \mathrm{mmol})$ at $0-5^{\circ} \mathrm{C}$. The mixture was stirred at $0-5^{\circ} \mathrm{C}$ for $30 \mathrm{~min}$, poured into ice-water $(20 \mathrm{ml})$, and extracted with $\mathrm{CH}_{2} \mathrm{Cl}_{2}(3 \times 20 \mathrm{ml})$. The extract was washed with water, dried, and evaporated. The residue was chromatographed (eluting with $\mathrm{CH}_{2} \mathrm{Cl}_{2}$ or ethyl acetate-hexane $(1: 19)$ ) to afford 3 (or 4 ).

3: Yield $99 \%$. mp $119-121{ }^{\circ} \mathrm{C}$ (ether). MS $m / z(\%): 413\left(\mathrm{M}^{+}, 100\right)$, 280 (71), 264 (16). Anal. Calcd for $\mathrm{C}_{18} \mathrm{H}_{14} \mathrm{~F}_{3} \mathrm{NO}_{5} \mathrm{~S}$ : C, 52.30; H, 3.41; $\mathrm{N}, 3.39$. Found: $\mathrm{C}, 52.34 ; \mathrm{H}, 3.44 ; \mathrm{N}, 3.34 .{ }^{1} \mathrm{H}-\mathrm{NMR} \delta: 3.46(3 \mathrm{H}, \mathrm{s}$, $\left.\mathrm{C}_{5}-\mathrm{OCH}_{3}\right), 4.04\left(3 \mathrm{H}, \mathrm{s}, \mathrm{C}_{8}-\mathrm{OCH}_{3}\right), 6.84\left(1 \mathrm{H}, \mathrm{d}, J=8.6 \mathrm{~Hz}, \mathrm{C}_{6}-\mathrm{H}\right), 7.09$ $\left(1 \mathrm{H}, \mathrm{s}, \mathrm{C}_{3}-\mathrm{H}\right), 7.12\left(1 \mathrm{H}, \mathrm{d}, J=8.6 \mathrm{~Hz}, \mathrm{C}_{7}-\mathrm{H}\right), 7.25-7.45\left(5 \mathrm{H}, \mathrm{m}, \mathrm{C}_{6} \mathrm{H}_{5}\right)$.

4: Yield $99 \%$. mp $73-74^{\circ} \mathrm{C}$ (ether-hexane). MS $m / z(\%): 457\left(\mathrm{M}^{+}\right.$, 100), 442 (43), 324 (53). Anal. Calcd for $\mathrm{C}_{20} \mathrm{H}_{18} \mathrm{~F}_{3} \mathrm{NO}_{6} \mathrm{~S}: \mathrm{C}, 52.52 ; \mathrm{H}$, 3.97; N, 3.06. Found: C, 52.44; H, 3.95; N, 3.01. ${ }^{1} \mathrm{H}-\mathrm{NMR} \delta: 2.41(3 \mathrm{H}$, s, $\left.\mathrm{C}_{7}-\mathrm{CH}_{3}\right), 3.19\left(3 \mathrm{H}, \mathrm{s}, \mathrm{C}_{5}-\mathrm{OCH}_{3}\right), 3.87\left(3 \mathrm{H}, \mathrm{s}, \mathrm{C}_{6}-\mathrm{OCH}_{3}\right), 4.07(3 \mathrm{H}$, $\left.\mathrm{s}, \mathrm{C}_{8}-\mathrm{OCH}_{3}\right), 6.97\left(1 \mathrm{H}, \mathrm{s}, \mathrm{C}_{3}-\mathrm{H}\right), 7.3-7.5\left(5 \mathrm{H}, \mathrm{m}, \mathrm{C}_{6} \mathrm{H}_{5}\right)$.

4-Phenylquinolines $(5,6)$ Formic acid $(0.11 \mathrm{ml}, 3 \mathrm{mmol})$ was added to a mixture of 3 (or 4) $(0.5 \mathrm{mmol})$, triethylamine $(0.56 \mathrm{ml}, 4 \mathrm{mmol})$, palladium acetate $(30 \mathrm{mg}, 0.13 \mathrm{mmol})$, and DPPF $(140 \mathrm{mg}, 0.25 \mathrm{mmol})$ 


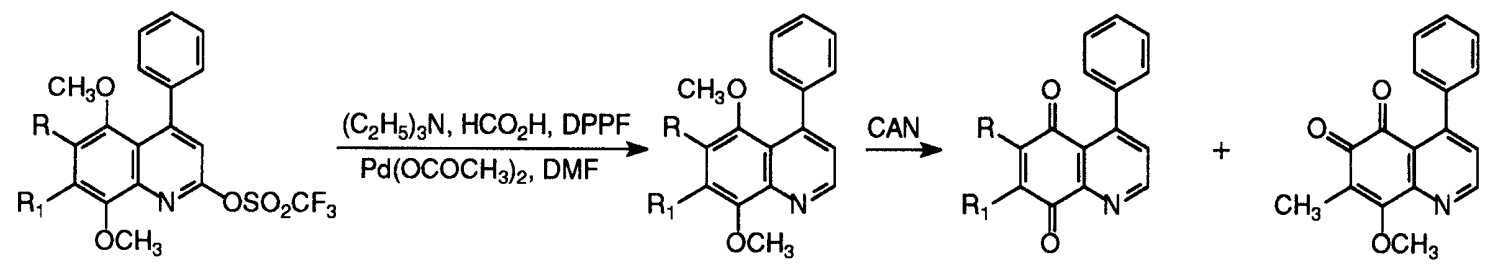

3: $\mathrm{R}=\mathrm{R}_{1}=\mathrm{H}$

4: $\mathrm{R}=\mathrm{OCH}_{3}, \mathrm{R}_{1}=\mathrm{CH}_{3}$ $\uparrow\left(\mathrm{CF}_{3} \mathrm{SO}_{2}\right)_{2} \mathrm{O}$

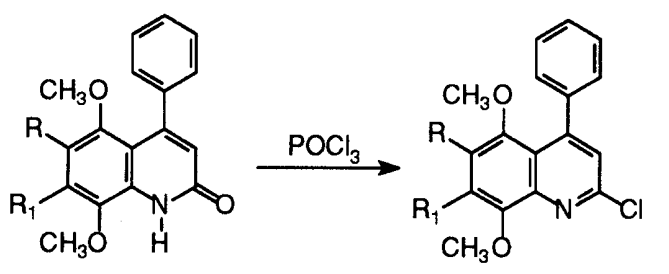

1: $\mathrm{R}=\mathrm{R}_{1}=\mathrm{H}$

2: $\mathrm{R}=\mathrm{OCH}_{3}, \mathrm{R}_{1}=\mathrm{CH}_{3}$

10: $R=R_{1}=H$

11: $\mathrm{R}=\mathrm{OCH}_{3}, \mathrm{R}_{1}=\mathrm{CH}_{3}$ $\mathrm{R}_{2}-\mathrm{NH}-\mathrm{R}_{3}$<smiles>[R]c1nc2c(N([R6])CCCCCC)cc(-c3ccccc3)c(OC)c2c(OC)c1[R]</smiles>

15a-d: $R=R_{1}=H$ 16a-d: $\mathrm{R}=\mathrm{OCH}_{3}, \mathrm{R}_{1}=\mathrm{CH}_{3}$<smiles>[R]C1=C([R])C(=O)c2c(-c3ccccc3)cc(Cl)nc2C1=O</smiles>

12: $\mathrm{R}=\mathrm{R}_{1}=\mathrm{H}$

13: $\mathrm{R}=\mathrm{OCH}_{3}, \mathrm{R}_{1}=\mathrm{CH}_{3}$<smiles>COC1=C(C)C(=O)C(=O)c2c(-c3ccccc3)cc(Cl)nc21</smiles>

14<smiles>[R]c1cc(OC)c2[nH]c(=O)cc([R2])c2c1O</smiles>

19a-d

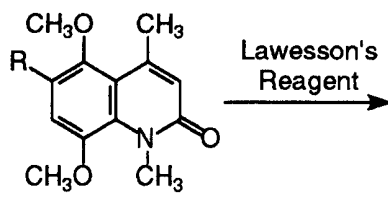

22a, b

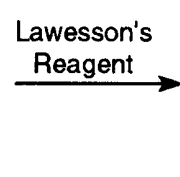

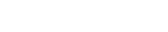<smiles>[R]c1cc(=S)[nH]c2c(OC)cc(Br)c(OC)c12</smiles>

20a-d<smiles>[2H]c1cc(OC)c2c(c(C)cc(=S)n2C)c1O</smiles>

23a, b<smiles>[R]C1=C([R])C(=O)c2c(-c3ccccc3)cc(NP)nc2C1=O</smiles>

17a-d: $R=\mathrm{R}_{1}=\mathrm{H}$ 18a-d: $R=\mathrm{OCH}_{3}, \mathrm{R}_{1}=\mathrm{CH}_{3}$

\author{
15-18 a: $R_{2}, R_{3}=-\left(\mathrm{CH}_{2}\right)_{5}$ \\ b: $R_{2}, R_{3}=-\left(\mathrm{CH}_{2}\right)_{4}-$ \\ c: $R_{2}, R_{3}=\longdiv { O }$ \\ d: $\mathrm{R}_{2}, \mathrm{R}_{3}=\mathrm{N}-\mathrm{CH}_{3}$
}<smiles>[R7]C1=CC(=O)c2nc(SSc3cc([R2])c4c(n3)C(=O)C=C([R7])C4=O)cc([R2])c2C1=O</smiles>

21a-d

Chart 1

in $N, N$-dimethylformamide $(5 \mathrm{ml})$. The mixture was stirred at $25^{\circ} \mathrm{C}$ for $1 \mathrm{~h}$, and then at $60^{\circ} \mathrm{C}$ for $2 \mathrm{~h}$. The reaction mixture was diluted with $1 \%$ $\mathrm{NaHCO}_{3}$ solution $(30 \mathrm{ml})$, and extracted with $\mathrm{CH}_{2} \mathrm{Cl}_{2}(3 \times 20 \mathrm{ml})$. The extract was washed with water, dried and evaporated. The residue was chromatographed (eluting with $\mathrm{CH}_{2} \mathrm{Cl}_{2}$-ethyl acetate $(19: 1)$ or ethyl acetate-hexane $(1: 4)$ ) to afford 5 (or 6 ).

5: Yield $99 \%$. mp $177-178^{\circ} \mathrm{C}\left(\mathrm{CH}_{2} \mathrm{Cl}_{2}\right.$-ether $)$. MS m/z (\%): 265 $\left(\mathrm{M}^{+}, 69\right), 250(100), 236(16), 220(20)$. Anal. Calcd for $\mathrm{C}_{17} \mathrm{H}_{15} \mathrm{NO}_{2}$ : C 76.96; H, 5.70; N, 5.28. Found: C, 76.90; H, 5.73; N, 5.23. ${ }^{1} \mathrm{H}-\mathrm{NMR} \delta$ : $3.45\left(3 \mathrm{H}, \mathrm{s}, \mathrm{C}_{5}-\mathrm{OCH}_{3}\right), 4.08\left(3 \mathrm{H}, \mathrm{s}, \mathrm{C}_{8}-\mathrm{OCH}_{3}\right), 6.77(1 \mathrm{H}, \mathrm{d}, J=8.6 \mathrm{~Hz}$ $\left.\mathrm{C}_{6}-\mathrm{H}\right), 7.00\left(1 \mathrm{H}, \mathrm{d}, J=8.6 \mathrm{~Hz}, \mathrm{C}_{7}-\mathrm{H}\right), 7.24\left(1 \mathrm{H}, \mathrm{d}, J=4.3 \mathrm{~Hz}, \mathrm{C}_{3}-\mathrm{H}\right)$, $7.25-7.45\left(5 \mathrm{H}, \mathrm{m}, \mathrm{C}_{6} \mathrm{H}_{5}\right), 8.91\left(1 \mathrm{H}, \mathrm{d}, J=4.3 \mathrm{~Hz}, \mathrm{C}_{2}-\mathrm{H}\right)$.

6: Yield $88 \% \cdot \mathrm{mp} 104-105^{\circ} \mathrm{C}$ (hexane). $\mathrm{MS} m / z(\%): 309\left(\mathrm{M}^{+}, 51\right)$, 294 (100). Anal. Calcd for $\mathrm{C}_{19} \mathrm{H}_{19} \mathrm{NO}_{3}: \mathrm{C}, 73.77 ; \mathrm{H}, 6.19 ; \mathrm{N}, 4.53$. Found: C, 73.53; H, 6.21; N, 4.48. ${ }^{1} \mathrm{H}-\mathrm{NMR} \delta: 2.43\left(3 \mathrm{H}, \mathrm{s}, \mathrm{C}_{7}-\mathrm{CH}_{3}\right), 3.19(3 \mathrm{H}$, $\left.\mathrm{s}, \mathrm{C}_{5}-\mathrm{OCH}_{3}\right), 3.87\left(3 \mathrm{H}, \mathrm{s}, \mathrm{C}_{6}-\mathrm{OCH}_{3}\right), 4.07\left(3 \mathrm{H}, \mathrm{s}, \mathrm{C}_{8}-\mathrm{OCH}_{3}\right), 7.13(1 \mathrm{H}$, $\left.\mathrm{d}, J=4.3 \mathrm{~Hz}, \mathrm{C}_{3}-\mathrm{H}\right), 7.3-7.5\left(5 \mathrm{H}, \mathrm{m}, \mathrm{C}_{6} \mathrm{H}_{5}\right), 8.82(1 \mathrm{H}, \mathrm{d}, J=4.3 \mathrm{~Hz}$, $\left.\mathrm{C}_{2}-\mathrm{H}\right)$.

2-Chloro-4-phenylquinolines (10, 11) A mixture of 4-phenyl-2(1H)quinolinone $(1,2)(1 \mathrm{mmol})$ and phosphorus oxychloride $(2 \mathrm{ml}, 21.5$ $\mathrm{mmol}$ ) was heated at $60-70^{\circ} \mathrm{C}$ for $30 \mathrm{~min}$. The reaction mixture was cooled, poured into ice-water $(50 \mathrm{ml})$, neutralized with $\mathrm{NaHCO}_{3}$, and 
Table 1. Analytical and Spectral Data for Quinolinequinones $(7-9,12-14,17,18,21,24)$

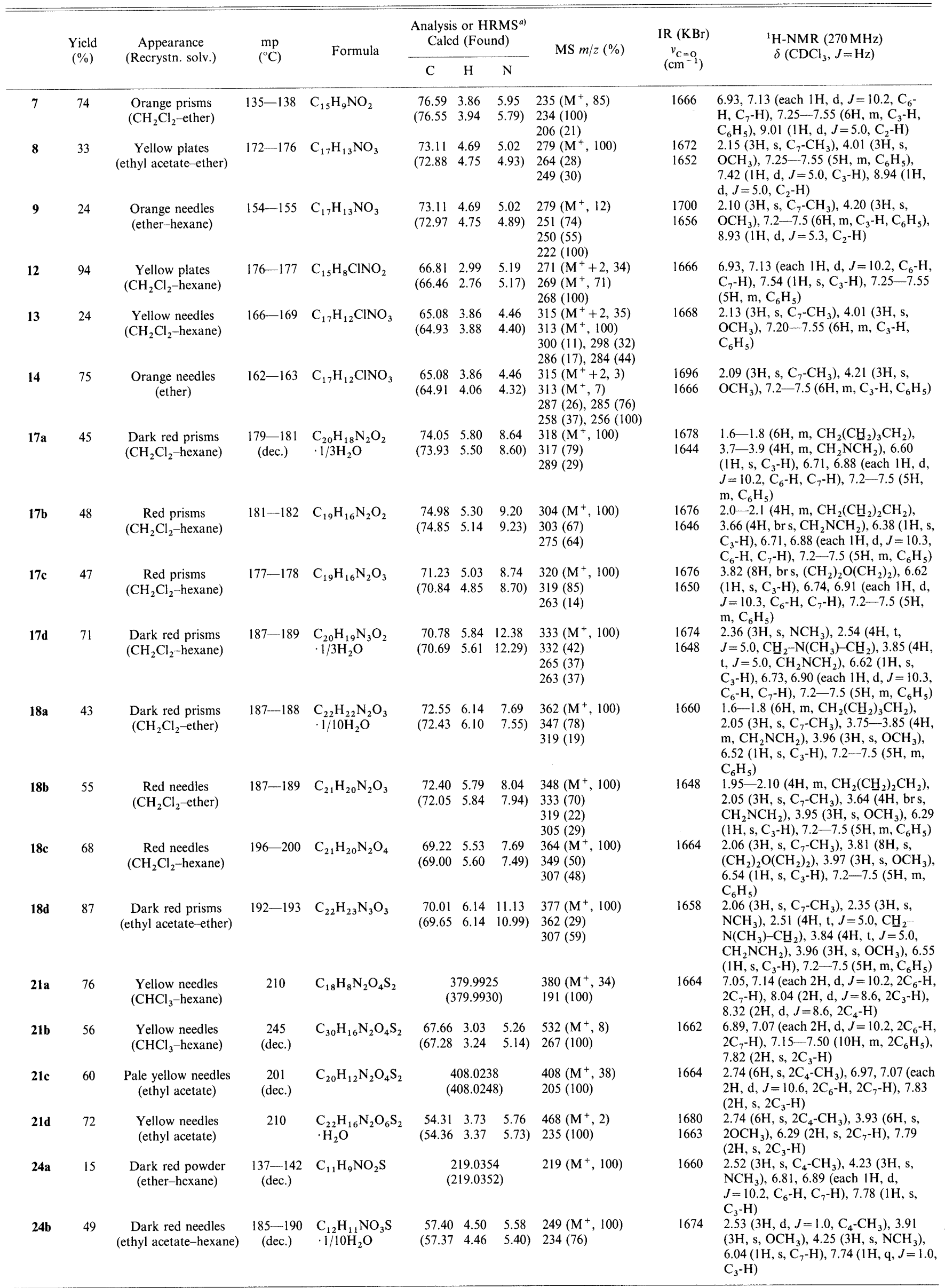

a) High-resolution MS. 
extracted with $\mathrm{CH}_{2} \mathrm{Cl}_{2}(3 \times 20 \mathrm{ml})$. The extract was washed with water, dried, and evaporated. The residue was chromatographed (eluting with ethyl acetate-hexane $(1: 9))$ to afford 2-chloro-4-phenylquinoline (10, 11).

10: Yield 99\%. mp 186-187 ${ }^{\circ} \mathrm{C}\left(\mathrm{CH}_{2} \mathrm{Cl}_{2}\right.$-hexane). MS $m / z(\%): 301$ $\left(\mathrm{M}^{+}+2,19\right), 299\left(\mathrm{M}^{+}, 57\right), 286(34), 284$ (100). Anal. Calcd for $\mathrm{C}_{17} \mathrm{H}_{14} \mathrm{ClNO}_{2}$ : C, 68.12; H, 4.71; N, 4.67. Found: C, 68.16; H, 4.51; N, 4.68. ${ }^{1} \mathrm{H}-\mathrm{NMR} \delta: 3.44\left(3 \mathrm{H}, \mathrm{s}, \mathrm{C}_{5}-\mathrm{OCH}_{3}\right), 4.05\left(3 \mathrm{H}, \mathrm{s}, \mathrm{C}_{8}-\mathrm{OCH}_{3}\right), 6.77$ $\left(1 \mathrm{H}, \mathrm{d}, J=8.6 \mathrm{~Hz}, \mathrm{C}_{6}-\mathrm{H}\right), 7.03\left(1 \mathrm{H}, \mathrm{d}, J=8.6 \mathrm{~Hz}, \mathrm{C}_{7}-\mathrm{H}\right), 7.2-7.5(6 \mathrm{H}$, $\mathrm{m}, \mathrm{C}_{3}-\mathrm{H}, \mathrm{C}_{6} \mathrm{H}_{5}$ ).

11: Yield $98 \%$. mp $131-133{ }^{\circ} \mathrm{C}$ (ether-hexane). MS $m / z(\%): 345$ $\left(\mathrm{M}^{+}+2,21\right), 343\left(\mathrm{M}^{+}, 57\right), 330$ (36), 328 (100). Anal. Calcd for $\mathrm{C}_{19} \mathrm{H}_{18} \mathrm{ClNO}_{3}$ : C, 66.38; H, 5.28; N, 4.07. Found: C, 66.25; H, 5.24; N, 4.03. ${ }^{1} \mathrm{H}-\mathrm{NMR} \delta: 2.41\left(3 \mathrm{H}, \mathrm{s}, \mathrm{C}_{7}-\mathrm{CH}_{3}\right), 3.18\left(3 \mathrm{H}, \mathrm{s}, \mathrm{C}_{5}-\mathrm{OCH}_{3}\right), 3.85$ $\left(3 \mathrm{H}, \mathrm{s}, \mathrm{C}_{6}-\mathrm{OCH}_{3}\right), 4.07\left(3 \mathrm{H}, \mathrm{s}, \mathrm{C}_{8}-\mathrm{OCH}_{3}\right), 7.12\left(1 \mathrm{H}, \mathrm{s}, \mathrm{C}_{3}-\mathrm{H}\right), 7.30-7.45$ $\left(5 \mathrm{H}, \mathrm{m}, \mathrm{C}_{6} \mathrm{H}_{5}\right)$.

2-Dialkylaminoquinolines $(15,16)$ A mixture of a 2-chloroquinoline $(10,11)(1 \mathrm{mmol})$ and 1-methylpiperazine (or piperidine, pyrrolidine, or morpholine; $2 \mathrm{ml}$ ) was refluxed for $2-3 \mathrm{~h}$, and then evaporated. The residue was chromatographed using ethyl acetate-methanol $(19: 1-7: 3)$ (or ethyl acetate-hexane $(1: 19-2: 3))$ as the eluent to afford 2-(4methyl-1-piperazinyl)quinoline (or 2-(1-piperidinyl)quinoline, 2-(1-pyrrolidinyl)quinoline, or 2-morpholinoquinoline; 15,16 ).

15a: Yield $99 \%$. mp $114-115^{\circ} \mathrm{C}\left(\mathrm{CH}_{2} \mathrm{Cl}_{2}\right.$-hexane). $\mathrm{MS} m / z(\%): 348$ $\left(\mathrm{M}^{+}, 100\right), 333$ (26), 319 (48), 265 (44). Anal. Calcd for $\mathrm{C}_{22} \mathrm{H}_{24} \mathrm{~N}_{2} \mathrm{O}_{2}$ : C, 75.83; H, 6.94; N, 8.04. Found: C, 76.05; H, 7.02; N, 7.99. ${ }^{1} \mathrm{H}-\mathrm{NMR}$ $\delta$ : $1.67\left(6 \mathrm{H}\right.$, brs, $\left.\mathrm{CH}_{2}\left(\mathrm{CH}_{2}\right)_{3} \mathrm{CH}_{2}\right), 3.37\left(3 \mathrm{H}, \mathrm{s}, \mathrm{C}_{5}-\mathrm{OCH}_{3}\right), 3.74(4 \mathrm{H}$, brs, $\left.\mathrm{CH}_{2} \mathrm{NCH}_{2}\right), 4.01\left(3 \mathrm{H}, \mathrm{s}, \mathrm{C}_{8}-\mathrm{OCH}_{3}\right), 6.44\left(1 \mathrm{H}, \mathrm{d}, J=8.6 \mathrm{~Hz}, \mathrm{C}_{6}-\mathrm{H}\right)$, $6.73\left(1 \mathrm{H}, \mathrm{s}, \mathrm{C}_{3}-\mathrm{H}\right), 6.88\left(1 \mathrm{H}, \mathrm{d}, J=8.6 \mathrm{~Hz}, \mathrm{C}_{7}-\mathrm{H}\right), 7.2-7.5(5 \mathrm{H}, \mathrm{m}$, $\mathrm{C}_{6} \mathrm{H}_{5}$ ).

15b: Yield $99 \%, \operatorname{mp~} 182-183^{\circ} \mathrm{C}\left(\mathrm{CH}_{2} \mathrm{Cl}_{2}\right.$-hexane). MS $m / z(\%): 334$ $\left(\mathrm{M}^{+}, 100\right), 319$ (54), 305 (37). Anal. Calcd for $\mathrm{C}_{21} \mathrm{H}_{22} \mathrm{~N}_{2} \mathrm{O}_{2}$ : C, 75.42; $\mathrm{H}, 6.63 ; \mathrm{N}, 8.38$. Found: C, 75.21; H, 6.60; N, 8.37. ${ }^{1} \mathrm{H}-\mathrm{NMR} \delta: 2.02$ $\left(4 \mathrm{H}\right.$, brs, $\left.\mathrm{CH}_{2}\left(\mathrm{CH}_{2}\right)_{2} \mathrm{CH}_{2}\right), 3.37\left(3 \mathrm{H}, \mathrm{s}, \mathrm{C}_{5}-\mathrm{OCH}_{3}\right), 3.65(4 \mathrm{H}$, brs, $\left.\mathrm{CH}_{2} \mathrm{NCH}_{2}\right), 4.02\left(3 \mathrm{H}, \mathrm{s}, \mathrm{C}_{8}-\mathrm{OCH}_{3}\right), 6.41\left(1 \mathrm{H}, \mathrm{d}, J=8.3 \mathrm{~Hz}, \mathrm{C}_{6}-\mathrm{H}\right), 6.49$ $\left(1 \mathrm{H}, \mathrm{s}, \mathrm{C}_{3}-\mathrm{H}\right), 6.89\left(1 \mathrm{H}, \mathrm{d}, J=8.6 \mathrm{~Hz}, \mathrm{C}_{7}-\mathrm{H}\right), 7.25-7.45\left(5 \mathrm{H}, \mathrm{m}, \mathrm{C}_{6} \mathrm{H}_{5}\right)$

15c: Yield $98 \%$. mp 139- $140^{\circ} \mathrm{C}\left(\mathrm{CH}_{2} \mathrm{Cl}_{2}\right.$-hexane). $\mathrm{MS} \mathrm{m/z}(\%): 350$ $\left(\mathrm{M}^{+}, 100\right), 319$ (53), 293 (42). Anal. Calcd for $\mathrm{C}_{21} \mathrm{H}_{22} \mathrm{~N}_{2} \mathrm{O}_{3}$ : C, 71.98; $\mathrm{H}, 6.33$; N, 7.99. Found: C, 71.87; H, 6.29; N, 7.98. ${ }^{1} \mathrm{H}-\mathrm{NMR} \delta: 3.39$ $\left(3 \mathrm{H}, \mathrm{s}, \mathrm{C}_{5}-\mathrm{OCH}_{3}\right), 3.74\left(4 \mathrm{H}, \mathrm{t}, J=5.0 \mathrm{~Hz}, \mathrm{CH}_{2} \mathrm{NCH}_{2}\right), 3.85(4 \mathrm{H}, \mathrm{t}$, $\left.J=5.0 \mathrm{~Hz}, \mathrm{CH}_{2} \mathrm{OCH}_{2}\right), 4.01\left(3 \mathrm{H}, \mathrm{s}, \mathrm{C}_{8}-\mathrm{OCH}_{3}\right), 6.49(1 \mathrm{H}, \mathrm{d}, J=8.6 \mathrm{~Hz}$, $\left.\mathrm{C}_{6}-\mathrm{H}\right), 6.71\left(1 \mathrm{H}, \mathrm{s}, \mathrm{C}_{3}-\mathrm{H}\right), 6.92\left(1 \mathrm{H}, \mathrm{d}, J=8.6 \mathrm{~Hz}, \mathrm{C}_{7}-\mathrm{H}\right), 7.2-7.5(5 \mathrm{H}$, $\mathrm{m}, \mathrm{C}_{6} \mathrm{H}_{5}$ ).

15d: Yield $99 \%$. mp $172-174^{\circ} \mathrm{C}\left(\mathrm{CH}_{2} \mathrm{Cl}_{2}\right.$-hexane). $\mathrm{MS} m / z(\%): 363$ $\left(\mathrm{M}^{+}, 7\right), 293$ (100), 280 (23), 263 (22). Anal. Calcd for $\mathrm{C}_{22} \mathrm{H}_{25} \mathrm{~N}_{3} \mathrm{O}_{2}: \mathrm{C}$, 72.70; H, 6.93; N, 11.56. Found: C, 72.49; H, 6.96; N, 11.55. ${ }^{1} \mathrm{H}-\mathrm{NMR}$ $\delta: 2.36\left(3 \mathrm{H}, \mathrm{s}, \mathrm{NCH}_{3}\right), 2.56\left(4 \mathrm{H}, \mathrm{t}, J=5.0 \mathrm{~Hz}, \mathrm{CH}_{2}-\mathrm{N}\left(\mathrm{CH}_{3}\right)-\mathrm{CH}_{2}\right), 3.38$ $\left(3 \mathrm{H}, \mathrm{s}, \mathrm{C}_{5}-\mathrm{OCH}_{3}\right), 3.80\left(4 \mathrm{H}, \mathrm{t}, J=5.0 \mathrm{~Hz}, \mathrm{CH}_{2} \mathrm{NCH}_{2}\right), 4.01$ ( $3 \mathrm{H}, \mathrm{s}$, $\left.\mathrm{C}_{8}-\mathrm{OCH}_{3}\right), 6.47\left(1 \mathrm{H}, \mathrm{d}, J=8.6 \mathrm{~Hz}, \mathrm{C}_{6}-\mathrm{H}\right), 6.73\left(1 \mathrm{H}, \mathrm{s}, \mathrm{C}_{3}-\mathrm{H}\right), 6.90(1 \mathrm{H}$, d, $\left.J=8.6 \mathrm{~Hz}, \mathrm{C}_{7}-\mathrm{H}\right), 7.2-7.5\left(5 \mathrm{H}, \mathrm{m}, \mathrm{C}_{6} \mathrm{H}_{5}\right)$

16a: Yield 94\%. mp $106-107^{\circ} \mathrm{C}$ (hexane). MS $m / z(\%): 392\left(\mathrm{M}^{+}\right.$, 100), 377 (67), 363 (18). Anal. Calcd for $\mathrm{C}_{24} \mathrm{H}_{28} \mathrm{~N}_{2} \mathrm{O}_{3}$ : C, 73.44; H, 7.19; N, 7.14. Found: C, 73.22; H, 7.25; N, 7.07. ${ }^{1} \mathrm{H}-\mathrm{NMR} \delta: 1.67(6 \mathrm{H}$, br s, $\left.\mathrm{CH}_{2}\left(\mathrm{CH}_{2}\right)_{3} \mathrm{CH}_{2}\right), 2.35\left(3 \mathrm{H}, \mathrm{s}, \mathrm{C}_{7}-\mathrm{CH}_{3}\right), 3.17\left(3 \mathrm{H}, \mathrm{s}, \mathrm{C}_{5}-\mathrm{OCH}_{3}\right), 3.72$ $\left(4 \mathrm{H}\right.$, brs, $\left.\mathrm{CH}_{2} \mathrm{NCH}_{2}\right), 3.78\left(3 \mathrm{H}, \mathrm{s}, \mathrm{C}_{6}-\mathrm{OCH}_{3}\right), 4.08\left(3 \mathrm{H}, \mathrm{s}, \mathrm{C}_{8}-\mathrm{OCH}_{3}\right)$, $6.70\left(1 \mathrm{H}, \mathrm{s}, \mathrm{C}_{3}-\mathrm{H}\right), 7.3-7.5\left(5 \mathrm{H}, \mathrm{m}, \mathrm{C}_{6} \mathrm{H}_{5}\right)$.

16b: Yield $96 \%$. mp 119-120 ${ }^{\circ} \mathrm{C}$ (hexane). MS $m / z(\%): 378\left(\mathrm{M}^{+}\right.$ 100), $363(90), 335$ (25). Anal. Calcd for $\mathrm{C}_{23} \mathrm{H}_{26} \mathrm{~N}_{2} \mathrm{O}_{3}$ : C, 72.99; H, 6.92; N, 7.40. Found: C, 72.89; H, 7.05; N, 7.25. ${ }^{1} \mathrm{H}-\mathrm{NMR} \delta: 1.95-2.10(4 \mathrm{H}$, $\left.\mathrm{m}, \mathrm{CH}_{2}\left(\mathrm{CH}_{2}\right)_{2} \mathrm{CH}_{2}\right), 2.35\left(3 \mathrm{H}, \mathrm{s}, \mathrm{C}_{7}-\mathrm{CH}_{3}\right), 3.16\left(3 \mathrm{H}, \mathrm{s}, \mathrm{C}_{5}-\mathrm{OCH}_{3}\right)$, 3.55-3.70 (4H, m, $\left.\mathrm{CH}_{2} \mathrm{NCH}_{2}\right), 3.77\left(3 \mathrm{H}, \mathrm{s}, \mathrm{C}_{6}-\mathrm{OCH}_{3}\right), 4.11(3 \mathrm{H}, \mathrm{s}$, $\left.\mathrm{C}_{8}-\mathrm{OCH}_{3}\right), 6.44\left(1 \mathrm{H}, \mathrm{s}, \mathrm{C}_{3}-\mathrm{H}\right), 7.30-7.45\left(5 \mathrm{H}, \mathrm{m}, \mathrm{C}_{6} \mathrm{H}_{5}\right)$.

16c: Yield $99 \%$. mp $154-156^{\circ} \mathrm{C}$ (ether). $\mathrm{MS} m / z(\%): 394\left(\mathrm{M}^{+}, 100\right)$ 379 (63). Anal. Calcd for $\mathrm{C}_{23} \mathrm{H}_{26} \mathrm{~N}_{2} \mathrm{O}_{4}: \mathrm{C}, 70.03 ; \mathrm{H}, 6.64 ; \mathrm{N}, 7.10$. Found: C, 70.03; H, 6.76; N, 6.91. ${ }^{1} \mathrm{H}-\mathrm{NMR} \delta: 2.36\left(3 \mathrm{H}, \mathrm{s}, \mathrm{C}_{7}-\mathrm{CH}_{3}\right), 3.17(3 \mathrm{H}$, $\left.\mathrm{s}, \mathrm{C}_{5}-\mathrm{OCH}_{3}\right), 3.70\left(4 \mathrm{H}, \mathrm{t}, J=5.0 \mathrm{~Hz}, \mathrm{CH}_{2} \mathrm{NCH}_{2}\right), 3.79\left(3 \mathrm{H}, \mathrm{s}, \mathrm{C}_{6}-\mathrm{OCH}_{3}\right)$, $3.85\left(4 \mathrm{H}, \mathrm{t}, J=5.0 \mathrm{~Hz}, \mathrm{CH}_{2} \mathrm{OCH}_{2}\right), 4.06\left(3 \mathrm{H}, \mathrm{s}, \mathrm{C}_{8}-\mathrm{OCH}_{3}\right), 6.67(\mathrm{IH}$, $\left.\mathrm{s}, \mathrm{C}_{3}-\mathrm{H}\right), 7.3-7.5\left(5 \mathrm{H}, \mathrm{m}, \mathrm{C}_{6} \mathrm{H}_{5}\right)$.

16d: Yield 96\%. oil. MS $m / z(\%)$ : $407\left(\mathrm{M}^{+}, 9\right), 337(100), 324(25)$, 307 (18). High-resolution MS Calcd for $\mathrm{C}_{24} \mathrm{H}_{29} \mathrm{~N}_{3} \mathrm{O}_{3}: 407.2209$. Found: 407.2209. ${ }^{1} \mathrm{H}-\mathrm{NMR} \delta: 2.35\left(6 \mathrm{H}, \mathrm{s}, \mathrm{C}_{7}-\mathrm{CH}_{3}, \mathrm{NCH}_{3}\right), 2.55(4 \mathrm{H}, \mathrm{t}$, $\left.J=5.0 \mathrm{~Hz}, \mathrm{CH}_{2}-\mathrm{N}\left(\mathrm{CH}_{3}\right)-\mathrm{CH}_{2}\right), 3.17\left(3 \mathrm{H}, \mathrm{s}, \mathrm{C}_{5}-\mathrm{OCH}_{3}\right), 3.76(4 \mathrm{H}, \mathrm{t}$, $\left.J=5.0 \mathrm{~Hz}, \mathrm{CH}_{2} \mathrm{NCH}_{2}\right), 3.78\left(3 \mathrm{H}, \mathrm{s}, \mathrm{C}_{6}-\mathrm{OCH}_{3}\right), 4.07\left(3 \mathrm{H}, \mathrm{s}, \mathrm{C}_{8}-\mathrm{OCH}_{3}\right)$, $6.69\left(1 \mathrm{H}, \mathrm{s}, \mathrm{C}_{3}-\mathrm{H}\right), 7.30-7.45\left(5 \mathrm{H}, \mathrm{m}, \mathrm{C}_{6} \mathrm{H}_{5}\right)$.
(4-Phenyl-)2(1H)-quinolinethiones (20a, b) Lawesson's reagent (809 $\mathrm{mg}, 2 \mathrm{mmol})$ was added to a solution of $2(1 \mathrm{H})$-quinolinone $(\mathbf{1 9 a}, \mathbf{b})$ $(2 \mathrm{mmol})$ in 1,2-dimethoxyethane $(40 \mathrm{ml})$. The resulting solution was refluxed for $1 \mathrm{~h}$, and then evaporated. The residue was chromatographed (eluting with $\mathrm{CH}_{2} \mathrm{Cl}_{2}$ ) to afford $20 \mathbf{a}, \mathbf{b}$.

20a: Yield $96 \%$. mp $212-214^{\circ} \mathrm{C}$ (ethyl acetate). MS $m / z(\%): 221$ $\left(\mathrm{M}^{+}, 100\right), 206$ (88), 191 (43). Anal. Calcd for $\mathrm{C}_{11} \mathrm{H}_{11} \mathrm{NO}_{2} \mathrm{~S}: \mathrm{C}, 59.71$; H, 5.01; N, 6.33. Found: C, 59.45; H, 5.11; N, 6.04. ${ }^{1} \mathrm{H}-\mathrm{NMR} \delta: 3.91$, 3.95 (each $\left.3 \mathrm{H}, \mathrm{s}, 2 \mathrm{OCH}_{3}\right), 6.59\left(1 \mathrm{H}, \mathrm{d}, J=8.6 \mathrm{~Hz}, \mathrm{C}_{6}-\mathrm{H}\right), 6.91(1 \mathrm{H}, \mathrm{d}$, $\left.J=8.6 \mathrm{~Hz}, \mathrm{C}_{7}-\mathrm{H}\right), 7.43\left(1 \mathrm{H}, \mathrm{dd}, J=9.2,1.3 \mathrm{~Hz}, \mathrm{C}_{3}-\mathrm{H}\right), 7.96(1 \mathrm{H}, \mathrm{d}$, $\left.J=9.2 \mathrm{~Hz}, \mathrm{C}_{4}-\mathrm{H}\right), 10.85(1 \mathrm{H}, \mathrm{br}, \mathrm{NH})$.

20b: Yield $94 \%$. mp $217-221^{\circ} \mathrm{C}$ (decomp.) $\left(\mathrm{CH}_{2} \mathrm{Cl}_{2}\right.$-hexane). MS $m / z(\%): 297\left(\mathrm{M}^{+}, 100\right), 282(83), 267(16)$. Anal. Calcd for $\mathrm{C}_{17} \mathrm{H}_{15} \mathrm{NO}_{2} \mathrm{~S}$ : C, 68.66; H, 5.08; N, 4.71. Found: C, 68.53; H, 4.94; N, 4.72. ${ }^{1} \mathrm{H}-\mathrm{NMR}$ $\delta: 3.41\left(3 \mathrm{H}, \mathrm{s}, \mathrm{C}_{5}-\mathrm{OCH}_{3}\right), 3.99\left(3 \mathrm{H}, \mathrm{s}, \mathrm{C}_{8}-\mathrm{OCH}_{3}\right), 6.58(1 \mathrm{H}, \mathrm{d}, J=8.6 \mathrm{~Hz}$, $\left.\mathrm{C}_{6}-\mathrm{H}\right), 6.97\left(1 \mathrm{H}, \mathrm{d}, J=8.6 \mathrm{~Hz}, \mathrm{C}_{7}-\mathrm{H}\right), 7.20-7.45\left(6 \mathrm{H}, \mathrm{m}, \mathrm{C}_{3}-\mathrm{H}, \mathrm{C}_{6} \mathrm{H}_{5}\right)$, $11.04(1 \mathrm{H}$, br, NH).

4-Methyl-2(1H)-quinolinethiones $(\mathbf{2 0 c}$, d) Lawesson's reagent $(2.0 \mathrm{~g}$, $5 \mathrm{mmol}$ ) was added to a solution of $2(1 \mathrm{H})$-quinolinone $(19 \mathbf{c}, \mathbf{d})(4 \mathrm{mmol})$ in 1,2-dimethoxyethane $(50 \mathrm{ml})$. The resulting solution was stirred at $25^{\circ} \mathrm{C}$ for $3 \mathrm{~h}$, and evaporated. The residue was chromatographed (eluting with $\mathrm{CH}_{2} \mathrm{Cl}_{2}-$ ethyl acetate $(3: 2)$ or $\left.\mathrm{CH}_{2} \mathrm{Cl}_{2}\right)$ to afford $20 \mathrm{c}$, d.

20c: Yield $99 \%$ mp $201{ }^{\circ} \mathrm{C}$ (ethyl acetate). $\mathrm{MS} m / z(\%): 235\left(\mathrm{M}^{+}\right.$, 94), 220 (100), 205 (37). Anal. Calcd for $\mathrm{C}_{12} \mathrm{H}_{13} \mathrm{NO}_{2} \mathrm{~S}: \mathrm{C}, 61.25 ; \mathrm{H}, 5.57$; $\mathrm{N}, 5.95$. Found: C, 61.29; H, 5.57; N, 5.91. ${ }^{1} \mathrm{H}-\mathrm{NMR} \delta: 2.64(3 \mathrm{H}, \mathrm{s}$, $\left.\mathrm{C}_{4}-\mathrm{CH}_{3}\right), 3.86,3.95\left(\right.$ each $\left.3 \mathrm{H}, \mathrm{s}, 2 \mathrm{OCH}_{3}\right), 6.61\left(1 \mathrm{H}, \mathrm{d}, J=8.9 \mathrm{~Hz}, \mathrm{C}_{6}-\mathrm{H}\right)$, $6.92\left(1 \mathrm{H}, \mathrm{d}, J=8.9 \mathrm{~Hz}, \mathrm{C}_{7}-\mathrm{H}\right), 7.27\left(1 \mathrm{H}, \mathrm{s}, \mathrm{C}_{3}-\mathrm{H}\right), 10.82(\mathrm{HH}, \mathrm{br}, \mathrm{NH})$.

20d: Yield $96 \%$. mp $213-216^{\circ} \mathrm{C}\left(\mathrm{CH}_{2} \mathrm{Cl}_{2}\right.$-ether). MS $m / z(\%): 265$ $\left(\mathrm{M}^{+}\right.$, 90), 250 (100). Anal. Calcd for $\mathrm{C}_{13} \mathrm{H}_{15} \mathrm{NO}_{3} \mathrm{~S}$ : C, 58.85; H, 5.70; $\mathrm{N}, 5.28$. Found: C, 58.66; H, 5.62; N, 5.28. ${ }^{1} \mathrm{H}-\mathrm{NMR} \delta: 2.66(3 \mathrm{H}, \mathrm{s}$, $\left.\mathrm{C}_{4}-\mathrm{CH}_{3}\right), 3.84,3.96,4.00\left(\operatorname{each} 3 \mathrm{H}, \mathrm{s}, 3 \mathrm{OCH}_{3}\right), 6.79\left(1 \mathrm{H}, \mathrm{s}, \mathrm{C}_{7}-\mathrm{H}\right), 7.28$ $\left(\mathrm{lH}, \mathrm{s}, \mathrm{C}_{3}-\mathrm{H}\right), 10.72(1 \mathrm{H}, \mathrm{br}, \mathrm{NH})$.

1,4-Dimethyl-2(1H)-quinolinethiones (23) Lawesson's reagent $(1.0 \mathrm{~g}$, $2.5 \mathrm{mmol})$ was added to a solution of $2(1 \mathrm{H})$-quinolinone $(22)(2 \mathrm{mmol})$ in 1,2-dimethoxyethane $(50 \mathrm{ml})$. The resulting solution was refluxed for $3 \mathrm{~h}$, and then evaporated. The residue was chromatographed (eluting with $\mathrm{CH}_{2} \mathrm{Cl}_{2}$ or ethyl acetate-hexane $\left.(1: 2)\right)$ to afford 23 .

23a: Yield $91 \%$. mp $136-139^{\circ} \mathrm{C}$ (ethyl acetate). MS $m / z(\%): 249$ $\left(\mathrm{M}^{+}, 100\right), 234$ (94), 219 (39). Anal. Calcd for $\mathrm{C}_{13} \mathrm{H}_{15} \mathrm{NO}_{2} \mathrm{~S}: \mathrm{C}, 62.62$; H, 6.06; N, 5.62. Found: C, 62.69; H, 6.05; N, 5.61. ${ }^{1} \mathrm{H}-\mathrm{NMR} \delta: 2.58$ $\left(3 \mathrm{H}, \mathrm{s}, \mathrm{C}_{4}-\mathrm{CH}_{3}\right), 3.85,3.87$ (each $\left.3 \mathrm{H}, \mathrm{s}, 2 \mathrm{OCH}_{3}\right), 4.25\left(3 \mathrm{H}, \mathrm{s}, \mathrm{NCH}_{3}\right)$, $6.73\left(1 \mathrm{H}, \mathrm{d}, J=8.9 \mathrm{~Hz}, \mathrm{C}_{6}-\mathrm{H}\right), 7.06\left(1 \mathrm{H}, \mathrm{d}, J=8.9 \mathrm{~Hz}, \mathrm{C}_{7}-\mathrm{H}\right), 7.47(1 \mathrm{H}$, $\left.\mathrm{s}, \mathrm{C}_{3}-\mathrm{H}\right)$

23b: Yield $91 \%$. mp $164-166^{\circ} \mathrm{C}$ (ethyl acetate). MS $m / z(\%): 279$ $\left(\mathrm{M}^{+}, 100\right), 264$ (65), 249 (19), 234 (22). Anal. Calcd for $\mathrm{C}_{14} \mathrm{H}_{17} \mathrm{NO}_{3} \mathrm{~S}$ : C, 60.19; H, 6.13; N, 5.01. Found: C, 60.01; H, 6.11; N, 4.97. ${ }^{1} \mathrm{H}-\mathrm{NMR}$ $\delta: 2.60\left(3 \mathrm{H}, \mathrm{d}, J=0.7 \mathrm{~Hz}, \mathrm{C}_{4}-\mathrm{CH}_{3}\right), 3.83,3.91,3.96\left(\right.$ each $\left.3 \mathrm{H}, \mathrm{s}, 3 \mathrm{OCH}_{3}\right)$, $4.24\left(3 \mathrm{H}, \mathrm{s}, \mathrm{NCH}_{3}\right), 6.85\left(1 \mathrm{H}, \mathrm{s}, \mathrm{C}_{7}-\mathrm{H}\right), 7.45\left(1 \mathrm{H}, \mathrm{q}, J=0.7 \mathrm{~Hz}, \mathrm{C}_{3}-\mathrm{H}\right)$.

Oxidative Demethylation of 5,8-Dimethoxy- and 5,6,8-Trimethoxy-4phenylquinolines $(5,6,10,11,15,16)$ Method A: A solution of CAN (1370 $\mathrm{mg}, 2.5 \mathrm{mmol})$ in acetonitrile-water $(1: 1,5 \mathrm{ml})$ was added dropwise to 4-phenylquinoline $(\mathbf{5}, \mathbf{1 1})(0.5 \mathrm{mmol})$ dissolved in acetonitrile-water $(4: 1,20 \mathrm{ml})$ containing pyridine-2,6-dicarboxylic acid $N$-oxide ${ }^{6)}(458 \mathrm{mg}$, $2.5 \mathrm{mmol}$ ) at $0-5^{\circ} \mathrm{C}$. The mixture was stirred at $0-5^{\circ} \mathrm{C}$ for $30 \mathrm{~min}$, diluted with water $(60 \mathrm{ml})$, and extracted with $\mathrm{CH}_{2} \mathrm{Cl}_{2}(4 \times 40 \mathrm{ml})$. The extract was washed with brine, dried and evaporated. The residue was chromatographed (eluting with ethyl acetate-hexane $(1: 9-3: 7)$ ) to afford $p$-quinone $(7,13)$ and/or $o$-quinone (14).

Method B: A solution of CAN (4.11 g, $7.5 \mathrm{mmol})$ in acetonitrile-water $(1: 1,20 \mathrm{ml})$ was added dropwise to 4 -phenylquinoline $(6)(157 \mathrm{mg}, 0.5$ $\mathrm{mmol})$ dissolved in acetonitrile-water $(4: 1,40 \mathrm{ml})$ containing pyridine2,6 -dicarboxylic acid $N$-oxide $(1373 \mathrm{mg}, 7.5 \mathrm{mmol})$ at $0-5^{\circ} \mathrm{C}$. The $\mathrm{mix}$ ture was stirred at $0-5^{\circ} \mathrm{C}$ for $30 \mathrm{~min}$, diluted with water $(100 \mathrm{ml})$, and extracted with $\mathrm{CH}_{2} \mathrm{Cl}_{2}(4 \times 40 \mathrm{ml})$. The extract was washed with brine, dried and evaporated. The residue was chromatographed. Elution with ethyl acetate-hexane $(3: 7)$ afforded the less polar $o$-quinone $(\mathbf{9}, 34 \mathrm{mg}$, $24 \%$ ), and further elution with ethyl acetate-hexane $(1: 1)$ afforded the more polar $p$-quinone (8, $46 \mathrm{mg}, 33 \%$ ).

Method C: A solution of CAN $(685 \mathrm{mg}, 1.25 \mathrm{mmol})$ in acetonitrilewater $(1: 1,5 \mathrm{ml})$ was added dropwise to 2-chloro-, 2-(1-piperidinyl)-, 2-(1-pyrrolidinyl)-, or 2-morpholinoquinoline $(10,15 \mathbf{a}-\mathbf{c}, 16 \mathbf{a}-\mathbf{c})$ $(0.5 \mathrm{mmol})$ dissolved in acetonitrile-water $(4: 1,10 \mathrm{ml})$ containing pyridine-2,6-dicarboxylic acid $N$-oxide $(229 \mathrm{mg}, 1.25 \mathrm{mmol})$ at $0-5^{\circ} \mathrm{C}$. The mixture was stirred at $0-5^{\circ} \mathrm{C}$ for $15-30 \mathrm{~min}$, diluted with water 
(60 ml), and extracted with $\mathrm{CH}_{2} \mathrm{Cl}_{2}(4 \times 40 \mathrm{ml})$. The extract was washed with brine, dried and evaporated. The residue was chromatographed (eluting with ethyl acetate-hexane $(1: 4-1: 1))$ to afford the corresponding $p$-quinone $(12,17 \mathbf{a}-\mathbf{c}, 18 \mathrm{a}-\mathrm{c})$.

Method D: A solution of CAN $(685 \mathrm{mg}, 1.25 \mathrm{mmol})$ in acetonitrilewater $(1: 1,5 \mathrm{ml})$ was added dropwise to 2 -(4-methyl-1-piperazinyl)quinoline (15d, 16d) $(0.5 \mathrm{mmol})$ dissolved in acetonitrile-water $(4: 1$, $10 \mathrm{ml})$ containing pyridine-2,6-dicarboxylic acid $N$-oxide $(229 \mathrm{mg}, 1.25$ $\mathrm{mmol}$ ) at $0-5^{\circ} \mathrm{C}$. The mixture was stirred at $0-5^{\circ} \mathrm{C}$ for $15-30 \mathrm{~min}$, diluted with water $(60 \mathrm{ml})$, neutralized with $\mathrm{NaHCO}_{3}$ and extracted with $\mathrm{CH}_{2} \mathrm{Cl}_{2}(4 \times 40 \mathrm{ml})$. The extract was washed with brine, dried and evaporated. The residue was chromatographed (eluting with ethyl acetate-methanol $(9: 1-7: 3))$ to afford the corresponding $p$-quinone (17d, 18d).

Oxidative Demethylation of $2(1 \mathrm{H})$-Quinolinethiones $(\mathbf{2 0}, \mathbf{2 3}) \quad$ Method A: A solution of CAN $(822 \mathrm{mg}, 1.5 \mathrm{mmol})$ in water $(6 \mathrm{ml})$ was added dropwise to a $2(1 \mathrm{H})$-quinolinethione $(\mathbf{2 0}, \mathbf{2 3 a})(0.5 \mathrm{mmol})$ dissolved in acetonitrile $(30 \mathrm{ml})$ at $0-5^{\circ} \mathrm{C}$. The mixture was stirred at $0-5^{\circ} \mathrm{C}$ for $30 \mathrm{~min}$, diluted with water $(100 \mathrm{ml})$, and extracted with $\mathrm{CH}_{2} \mathrm{Cl}_{2}(3 \times$ $100 \mathrm{ml}$ ). The extract was washed with brine, dried and evaporated. The residue was chromatographed (eluting with $\mathrm{CH}_{2} \mathrm{Cl}_{2}$-methanol (99:1, 21a), ethyl acetate-hexane (3:7-1:1, 21b), $\mathrm{CHCl}_{3}$ acetone $(49: 1,21 \mathbf{c})$, $\mathrm{CH}_{2} \mathrm{Cl}_{2}$-ethyl acetate $(17: 3, \mathbf{2 1 d})$, and $\mathrm{CHCl}_{3}$-acetone $\left.(99: 1,24 a)\right)$ to afford the corresponding $p$-quinone $(\mathbf{2 1}, \mathbf{2 4 a})$.

Method B: Nitric acid $(6 \mathrm{~N}, 7.5 \mathrm{ml})$ and $\mathrm{AgO}(248 \mathrm{mg}, 2 \mathrm{mmol})$ were added to a solution of $\mathbf{2 3 b}(56 \mathrm{mg}, 0.2 \mathrm{mmol})$ in 1,4-dioxane $(15 \mathrm{ml})$. The mixture was stirred at $25^{\circ} \mathrm{C}$ for $1 \mathrm{~h}$, diluted with water $(100 \mathrm{ml})$, and extracted with $\mathrm{CH}_{2} \mathrm{Cl}_{2}(3 \times 100 \mathrm{ml})$. The extract was washed with brine, dried and evaporated. The residue was chromatographed (eluting with $\mathrm{CHCl}_{3}$-acetone $\left.(99: 1)\right)$ to afford $24 \mathrm{~b}(24 \mathrm{mg}, 49 \%)$.

Acknowledgements We thank Mr. N. Eguchi, Ms. T. Koseki, and Ms. S. Yoshioka in the Analytical Center of our College for measurement of spectral data (MS) and microanalytical data.

\section{References}

1) a) Take Y., Inouye Y., Nakamura S., Allaudeen H. S., Kubo A., J. Antibiot., 42, 107-115 (1989); b) Inouye Y., Take Y., Oogose K., Kubo A., Nakamura S., ibid., 40, 105-107 (1987).

2) a) Take Y., Oogose K., Kubo T., Inouye Y., Nakamura S., Kitahara Y., Kubo A., J. Antibiot., 40, 679-684 (1987); b) Inouye Y., Matsumoto H., Morishige R., Kitahara Y., Kubo A., Nakamura S., Chem. Pharm. Bull., 39, 994-998 (1991).

3) a) Kitahara Y., Nagaoka Y., Matsumura T., Kubo A., Heterocycles, 38, 659-678 (1994); b) Kitahara Y., Yonezawa T., Kubo A., ibid., 38, 1919-1926 (1994); c) Kitahara Y., Nakahara S., Shimizu M., Yonezawa T., Kubo A., ibid., 36, 1909-1924 (1993); d) Kitahara Y., Nakai T., Shimizu M., Kubo A., Chem. Pharm. Bull., 38, 2841-2846 (1990); e) Kitahara Y., Nakai T., Nakahara S., Akazawa M., Shimizu M., Kubo A., ibid., 39, 2256-2263(1991).

4) Cacchi S., Ciattini P. G., Morera E., Ortar G., Tetrahedron Lett., 27, 5541-5544 (1986).

5) Snyder C. D., Rapoport H., J. Am. Chem. Soc., 94, 227-231 (1972).

6) Syper L., Kloc K., Mlochowski J., Szulc Z., Synthesis, 1979, $521-522$. 